\title{
Leidenforst gas ratchets driven by thermal creep
}

\author{
Alois Würger \\ Laboratoire Ondes et Matière d'Aquitaine, Université de Bordeaux $\&$ CNRS, \\ 351 cours de la Libération, 33405 Talence, France
}

\begin{abstract}
We show that thermal creep is at the origin of the recently discovered Leidenfrost ratchet, where liquid droplets float on a vapor layer along a heated saw-tooth surface and accelerate to velocities of up to $40 \mathrm{~cm} / \mathrm{s}$. As the active element, the asymmetric temperature profile at each ratchet summit rectifies the vapor flow in the boundary layer. This mechanism works at low Reynolds number and provides a novel tool for controlling gas flow at nanostructured surfaces.

PACS numbers: 47.61.-k, 47.15.Rq, 44.20.+b, 68.03.-g
\end{abstract}

PACS numbers:

Liquid spilled on a hot surface rapidly evaporates. At the Leidenfrost temperature well above the boiling point, however, one observes long-lived droplets that levitate due to the excess pressure resulting from the permanent feed of vapor at the bottom. Their contact-free suspension makes such Leidenfrost droplets very mobile. Linke et al. observed that, when placed on a millimeter-sized brass ratchet, the droplets rapidly accelerate to a speed of about $10 \mathrm{~cm} / \mathrm{s}$ [1]. Very recently, Lagubeau et al. found that the same effect occurs for a piece of solid dry ice, and thus is not related to properties of the liquid phase [2. Even more surprisingly, Ok et al. reported that reducing the ratchet profile to $200 \mathrm{~nm}$, has little effect on the droplet velocity [3].

Unlike other self-propulsion mechanisms based on chemical or thermal gradients 48, this motion is not directed along an applied field but rather arises from the asymmetric surface structure of the solid support. This sawtooth profile acts as a rectifier transferring momentum on the interstitial vapor; the resulting gas flow advects the floating Leidenfrost droplet. The experimental findings [1 3] suggest that there is a common principle that works for both liquids and solids, and independently of the height of the ratchet profile. Several ideas have been put forward, relying on non-uniform Laplace pressure and Marangoni forces in the droplet, surface vibrations, or rectification of the radial vapor flow through the non-linear term of the Navier-Stokes equation [1 3, 9]; yet none of them explains all of the mentioned experiments. In particular, the submicron ratchets of Ok et al. 3] exclude non-linear hydrodynamics as the dominant mechanism, as illustrated in the left panel of Fig. 1: For small profile $D$, the gas velocity and the effective Reynolds number Re in the ratchet layer are much smaller than at midheight where $\operatorname{Re} \sim 1$ [2]; thus rectification is expected to disappear for $D \ll h_{0}$, whereas the data of Ok et al. rather show the opposite behavior.

In his 1879 attempt to explain Crookes' radiometer experiment and building on Reynolds' theory for thermal transpiration [10, Maxwell showed the existence of thermal creep velocity along a solid-gas interface [11,

$$
v_{C}=\frac{3}{4} \nu \frac{\nabla_{\|} T}{T}
$$
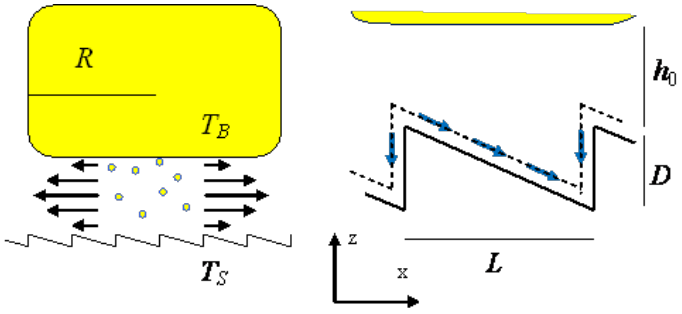

FIG. 1: Leidenfrost droplet on a ratchet. The left panel shows the gas flow due to the evaporation at the bottom of a droplet of radius $R$; the temperature of the solid $T_{S}$ is significantly above the droplet's boiling temperature $T_{B}$. The arrows in the right panel indicate the thermal creep flow along the ratchet of period $L$ and height $D$. The dashed line indicates the boundary layer; its thickness $\ell$ corresponds to the molecular mean free path. In recent experiments the ratchet height $D$ varies from $200 \mathrm{~nm}$ to about $1 \mathrm{~mm}$; for small $D$ one has $h_{0}=10 \ldots 100 \mu \mathrm{m}$.

where $\nu$ is the kinematic viscosity of the vapor and $\nabla_{\|} T$ the parallel component of the temperature gradient. Kinetic theory relates this gas flow to the non-uniform density and velocity distribution: Molecules coming from the cold side and hitting the surface at a given point, are more frequent but carry lower momentum than those from the hot side, thus resulting in an off-diagonal component of the surface stress and the boundary velocity $v_{C}$ [1]. Thermal creep drives aerosol thermophoresis [12, repels air-suspended particles from a hot surface [13, and operates in small-scale gas flow devices such as thermally actuated microcantilevers and Knudsen pumps [14 19].

The present Letter points out the role of thermal creep for self-propelling Leidenfrost droplets and, in particular, analyzes the flow around a ratchet summit. The essential argument is illustrated in the right panel of Fig. 1. In the cleft below the droplet, there is a strong temperature gradient of several tens of Kelvin per micron. Because of the asymmetric profile, the horizontal component of the creep velocity has a finite mean value; the resulting gas flow along the ratchet surface drags the droplet toward the right. Note that this argument does not rely on the existence of the outward gas flow illustrated in the left panel of Fig. 1. 
Our detailed analysis relies on Stokes hydrodynamics. In anology to thermal transport in colloidal dispersions [20, 21], the droplet velocity is derived from the overall force balance on a closed surface. In the absence of external forces in horizontal direction one has

$$
\oint \sigma_{x n} d S=0
$$

where $\sigma_{x n}$ is the stress pulling in $x$-direction on the area element $d S$ with normal $n$. The stress tensor $\sigma_{i j}=\sigma_{i j}^{\prime}-$ $P \delta_{i j}$ comprises a viscous part $\sigma_{i j}^{\prime}=\eta\left(\partial_{i} v_{j}+\partial_{j} v_{i}\right)$ and the excess pressure $P$. A non-uniform flow velocity $v$ in the cleft of width $h$ creates a stress of the order $\eta v / h$, which by far exceeds the viscous drag at the remaining part of the droplet surface $\sim \eta v / R$. Thus the surface integral may be limited to the part between droplet and support; it closely follows the ratchet profile beyond the boundary layer, as indicated by the dashed line in Fig. 1.

The velocity profile in the cleft comprises two contributions of different origin. The first one, due to evaporation at the bottom of the droplet, is the outside gas flow in the left panel of Fig. 1; in the framework of Stokes hydrodynamics it does not contribute to the stress integral. Thus in the following, we consider the second velocity term, which arises from the thermal creep along the ratchet profile, as indicated by the arrows in the right panel.

The rectification mechanism is most obvious when comparing the viscous stress at the two slopes of the ratchet. The normal on the vertical part points in $x$ direction; the corresponding diagonal element $\sigma_{x x}^{\prime}=$ $2 \eta d v_{x} / d x$ vanishes since $v_{x}$ and its derivative are zero. On the opposite side of slope $m=D / L$, the stress $\sigma_{x n}^{\prime}$ is finite. The hydrostatic pressure varies little along the profile and will be discarded; then Eq. 22 reduces to the condition

$$
\left\langle\sigma_{x z}^{\prime}\right\rangle=\frac{1}{L} \int_{0}^{L} \sigma_{x z}^{\prime} d x=0
$$

on the viscous drag on the slope of the ratchet tooth. If the droplet is immobile, the shear stress reads as $\sigma_{x n}^{\prime} \approx$ $-\eta v_{C} / h$, where $h$ is the width of the cleft. In order to satisfy (3) the droplet moves at a velocity $u$, leading to $\sigma_{x z}^{\prime}=\eta\left[u-v_{C}(x)\right] / h$. Inserting $\sigma_{x z}^{\prime}$ in (3) one readily obtains the expression for the drift velocity,

$$
u=\frac{\left\langle v_{C} / h\right\rangle}{\langle 1 / h\rangle} \text {. }
$$

The temperature profile is determined by the boundary conditions at the solid-gas interface, imposing continuous temperature and heat flow through the interface. Because of the important difference in thermal conductivity of the brass support and the vapor layer, $\kappa_{V} / \kappa_{S} \sim 10^{-4}$, the temperature profile is strongly distorted, and the gradient is much larger in the vapor phase.

For a first estimate we calculate $\nabla T_{\|}$far from the corners, in the middle part of a ratchet tooth. In the

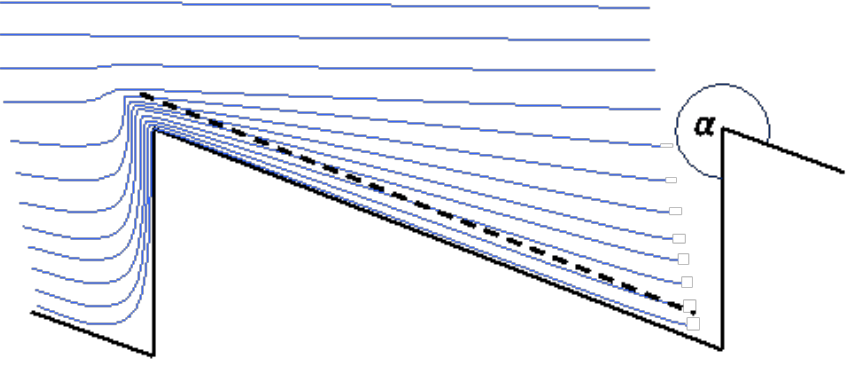

FIG. 2: Temperature profile close to a solid-vapor interface. Due to the small conductivity ratio $\kappa_{V} / \kappa_{S}$, the isotherms (solid lines) are strongly distorted. At a distance of one molecular mean free path from the hot surface (dashed line), the temperature gradient in the vapor has a significant component parallel to the surface, $\nabla T_{\|}$, which is largest close to the upper corner.

limit $\kappa_{V} / \kappa_{S} \rightarrow 0$ the brass surface is at constant temperature $T_{S}$, and the profile in the cleft is given by $T(x, z)=T_{B}-(z / h) \Delta T$, where $\Delta T=T_{S}-T_{B}$ and $h=h_{0}+x D / L$. The resulting temperature gradient is perpendicular on the solid-vapor and droplet-vapor interfaces. The velocity distribution of the molecules hitting the brass surface is given by the temperature profile evaluated at one mean-free path from the brass surface, $z=\ell-h$. At this finite distance, the gradient has a component parallel to the surface $\nabla T_{\|} \sim \Delta T \ell / h^{2}$. Replacing $h$ with $h_{0}$ and discarding numerical factors gives a rough estimate for the drift velocity,

$$
u \sim \nu \ell / h_{0}^{2}
$$

With the mean-free path $\ell=130 \mathrm{~nm}$ and the kinematic viscosity $\nu=60 \mathrm{~mm}^{2} / \mathrm{s}$ of vapor at $300^{\circ} \mathrm{C}$, and $h_{0} \sim$ $10 \mu \mathrm{m} 22$, one finds $u \sim 10 \mathrm{~cm} / \mathrm{s}$, which is in qualitative agreement with experiment [1].

For the sake of a more quantitative description we refine the vapor temperature profile close to the upper corner of the ratchet, which turns out to dominate the creep flow. In analogy to the electrostatic potential of a charged polygon, a simple conformal transformation provides the expression $T(r, \varphi)=T_{S}-$ $\Delta T\left(r / h_{0}\right)^{\pi / \alpha} \sin (\pi \varphi / \alpha)$ [24], where $r, \varphi$ are polar coordinates with respect to the corner. The angle $\alpha$ is related to the aspect ratio $m=D / L=-\cot \alpha$; for the ratchets of Ref. [3] one finds the exponent $\pi / \alpha \approx 0.63$. The resulting parallel component of the gradient along the dashed line close to point $A$ reads 23 .

$$
\nabla T_{\|}=\hat{\xi} \Delta T \frac{\ell h_{0}^{-\pi / \alpha}}{r^{2-\pi / \alpha}}
$$

where $\hat{\xi}=-\frac{\pi^{2}}{\alpha^{2}} \cos \frac{\alpha}{2}$. Its essential feature is the weak singularity at the ratchet summit, very similar to the electric field close to a charged cusp. The molecular mean-free path $\ell$ provides a physical cut-off for the divergency.

Now the drift velocity is evaluated in terms of Eq. (4), 


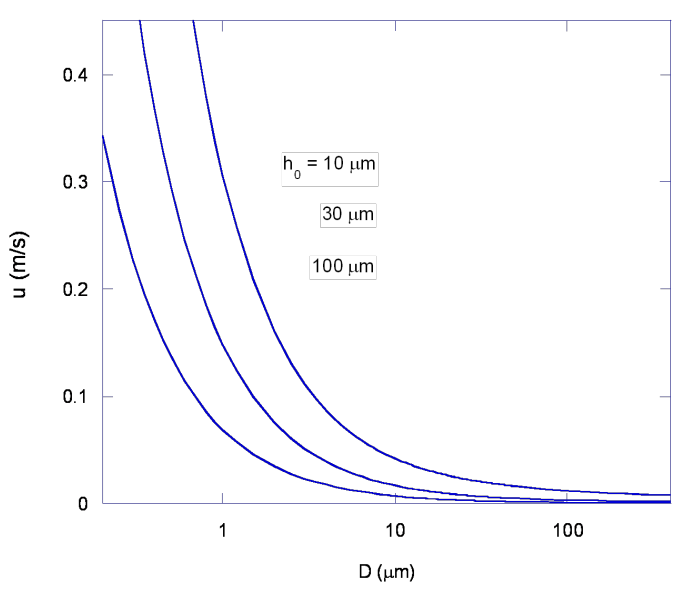

FIG. 3: Drift velocity $u$ as a function of the ratchet parameter $D$ for $h_{0}=10,30,100 \mu \mathrm{m}$. The curves are calculated from Eq. 77. with $\nu=60 \mathrm{~mm}^{2} / \mathrm{s}, \ell=130 \mathrm{~nm}, \pi / \alpha=0.63, L / D=4$, and $\Delta T / T=1 / 2$.

resulting in 23]

$$
u=\xi \frac{\Delta T}{T_{S}} \frac{\nu}{h_{0}}\left(\frac{\ell}{h_{0}}\right)^{\pi / \alpha} \frac{D / L}{\ln \left(1+D / h_{0}\right)}
$$

with the numerical prefactor $\xi \approx 0.6[23]$. This expression confirms the estimate (5) yet shows additional dependencies on the ratchet parameters. Fig. 3 reveals a striking variation of $u$ with $D$ : the smaller the ratchet profile, the larger the droplet velocity. This at first sight counterintuitive result is confirmed by the experiment of Ok et al. 3]: Their data at intermediate temperatures are well fitted by a logarithmic variation, similar to (7). Though this comparison does not account for the implicit dependence of $h_{0}$ on $D$, one may safely conclude on a qualitative agreement of $(7)$ with the data. Note that for a ratchet driven by non-linear hydrodynamics, one expects the opposite behavior, i.e., a smaller velocity for small $D$. Indeed, from the left panel of Fig. 1 it is clear that for $D \ll h_{0}$, the gas velocity in the ratchet layer is small and the non-linear term $(\mathbf{v} \cdot \boldsymbol{\nabla}) \mathbf{v}$ of the Navier-Stokes equation insignificant.

So far we have considered liquid droplets on a ratchet. The same mechanism holds for a piece of dry ice (solid $\mathrm{CO}_{2}$ ) above its sublimation temperature floating above a hot metal surface; when graving a saw tooth profile at its lower face, Lagubeau et al. observed motion similar to the droplets discussed so far. Since the creep velocity occurs at the bottom of the dry ice, the mean velocity of the vapor in the cleft is zero, as illustrated in Fig. 4.

Lagubeau et al. measured the force $F$ which required to immobilize a droplet floating on a ratchet [2. In the range $R=1 \ldots 7 \mathrm{~mm}$, they found values from 3 to 30 microNewton, and a power law $F \propto R^{\beta}$, with an expo-

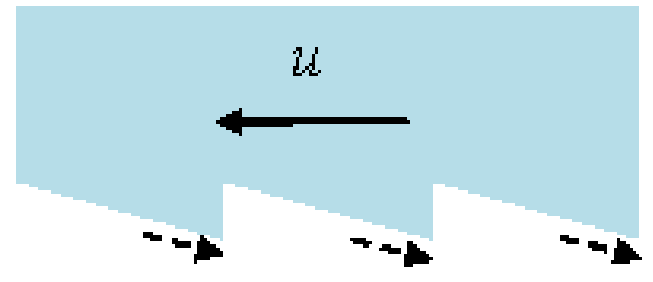

hot

FIG. 4: Thermal creep below a piece of dry ice ( solid $\mathrm{CO}_{2}$ ) floating above a hot metal surface due to sublimation [2]. Due to the ratchet profile printed at its lower side, there is a parallel temperature gradient $\nabla T_{\|}$as indicated by dashed arrows; the rectified thermal creep flow propels the disk to the left. The mean velocity of the vapor in the cleft is zero.

nent $\beta \approx 1.5$. In the present work, this force is given by the integral of the shear stress over the contact area, $F=\pi R^{2} \eta\left\langle v_{C} / h\right\rangle$. With the above expression for the thermal creep velocity one finds

$$
F=\pi \xi \frac{\eta R^{2} D}{L^{2}} \nu \frac{\Delta T}{T} \frac{\ell^{\pi / \alpha}}{h_{0}^{1+\pi / \alpha}} .
$$

With the relation $h_{0} \propto \sqrt{R}[2$, the force varies with the droplet size as $\beta=\frac{3}{2}-\frac{\pi}{2 \alpha} \approx 1.2$; within the experimental uncertainities, this compares favorably with the measured value.

The thermal-creep mechanism described here is not limited to the motion of Leidenfrost droplets. As a straightforward application we discuss the gas pump shown in Fig. 5, which consists of two nanostructured plates at temperatures differing by $\Delta T$. Both solid interfaces show thermal creep and thus impose a unifom gas flow across the cleft. For a sufficiently small ratchet profile, $D<h_{0}$, Eq. (7) simplifies to

$$
u_{0}=\xi \frac{\nu}{L} \frac{\Delta T}{T}\left(\frac{\ell}{h_{0}}\right)^{\pi / \alpha}
$$

This velocity may attain several meters per second. It turns out instructive to compare this ratchet with a Knudsen pump; in the present case, the thermal gradient is perpendicular to the gas flow, whereas both are parallel in the latter. Moreover, a Knudsen pump requires the system size to be comparable to or smaller than the mean-free path, and thus is restricted to very dilute gases. Although the ratchet mechanism does depend on the ratio $\ell / h_{0}$, it works for films that are hundred times thicker than the mean free path. 


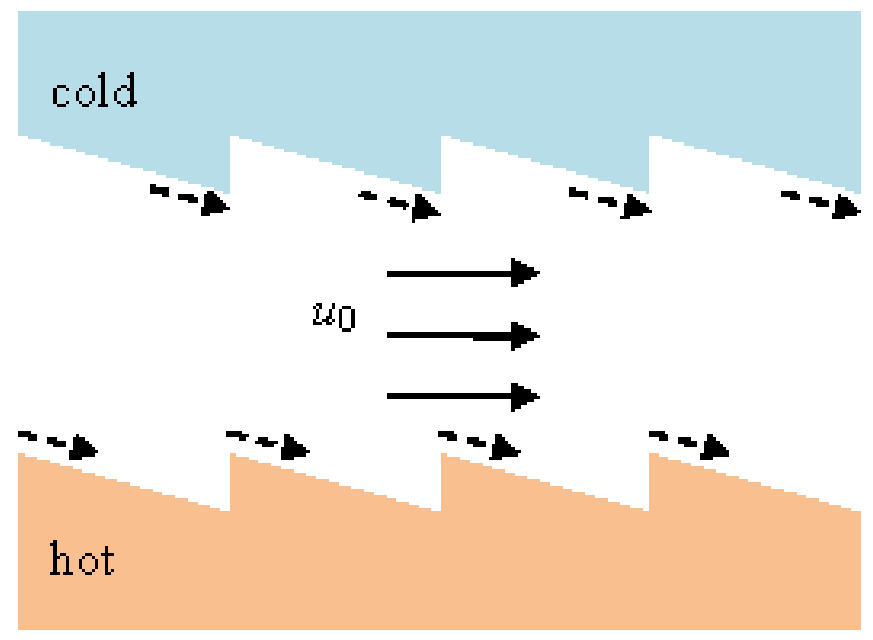

FIG. 5: Gas pump driven by thermal creep. The thermal gradient across the channel is given by their temperature difference $\Delta T$ and spacing $h_{0}$. At the ratchet corners, there is a parallel component $\nabla T_{\|}$, as indicated by dashed arrows. Thermal creep gives rise to a uniform gas flow at velocity $u_{0}$, as given in Eq. (4). Note the opposite orientation of the saw teeth on the cold and hot side.

[1] H. Linke, et al, Phys. Rev. Lett. 96, 154502 (2006)

[2] G. Lagubeau, M. Le Merrer, C. Clanet, D. Quéré, Nature Physics 7, 395 (2011)

[3] J. T. Ok, E. Lopez-Oña, D.E. Nikitopoulos, H. Wong, S. Park, Microfluid Nanofluid 10, 1045 (2011)

[4] F. Brochard, Langmuir 5, 432 (1989)

[5] A.M. Cazabat, F. Heslot, S.M. Troian, P. Carles, Nature 346, 824 (1990)

[6] P.-G. de Gennes, F. Brochard-Wyart, D. Quéré, Capillarity and Wetting Phenomena (Springer, NewYork, 2003).

[7] M. K. Chaudhury and G. M. Whitesides, Science 256, 1539 (1992).

[8] K. Ichimura, S.-K. Oh, and M. Nakagawa, Science 288, $1624(2000)$

[9] D. Quéré, A. Ajdari, Nature Mater. 5, 429 (2006)

[10] O. Reynolds, Phil. Trans. R. Soc. 170, 727 (1879)

[11] J.C. Maxwell, Phil. Trans. R. Soc. 170, 231 (1879)

[12] B. V. Derjaguin, Yu. Yalamov, J. Coll. Sci. 20, 555 (1965)

[13] L. Talbot, R.K. Cheng, R.W. Schefer, D.R. Willis, J. Fluid Mech. 101, 737 (1980)

[14] S.E. Vargo, E.P. Muntz, G.R. Shiflett, W.C. Tang, J.
Vac. Sci. Technol. A 17, 2306 (1999)

[15] A. Passian, R. J.Warmack, T. L. Ferrell, T. Thundat, Phys. Rev. Lett. 90, 124503 (2003)

[16] S. Colin, Microfluid. Nanofluid. 1, 268 (2005)

[17] B. Gotsmann, U. Dürig, Appl. Phys. Lett. 87, 194102 (2005)

[18] S. McNamara, Y.B. Gianchandani, J. Microelectromech. Syst. 14, 741 (2005)

[19] N.K. Gupta, Y.B. Gianchandani, Appl. Phyś. Lett. 93, 193511 (2008)

[20] R. Piazza, Soft Matter 4, 1740 (2008)

[21] A. Würger, Rep. Prog. Phys. 73, 126601 (2010)

[22] A.-L. Biance, C. Clanet, D. Quéré, Phys. Fluids 15, 1632 (2003)

[23] Supplemental material

[24] T.A. Driscoll, L.N. Trefethen, Schwarz-Christoffel mapping, Cambridge University Press, (Cambridge 2002)

[25] M. Knudsen, Ann. Phys. (Leipzig) 31, 205 (1910) and 33, 1435 (1910) 